\title{
Organizations Related to the Study of Political Science
}

*These organizations did not respond to our survey, and information is reprinted from previous editions of $P S$.

\begin{tabular}{|c|c|c|c|}
\hline Organization & Officers & Publications & Meetings \\
\hline $\begin{array}{l}\text { *AMERICAN } \\
\text { ANTHROPOLOGICAL } \\
\text { ASSOCIATION } \\
1703 \text { New Hampshire Ave., } \\
\text { NW, Washington, DC } 20009 \\
\text { Phone: (202) 232-8800 } \\
\text { Fax: (202) 667-5345 }\end{array}$ & President: Annette Weiner & $\begin{array}{l}\text { Special Publications } \\
\text { Guide to Departments of } \\
\text { Anthropology } \\
\text { American Anthropologist, Janet } \\
\text { Keller, Editor-in-Chief } \\
\text { Anthropology Newsletter, David B. } \\
\text { Givens, Editor } \\
\text { American Ethnologist, Donald } \\
\text { Brenneis, Editor } \\
\text { Central Issues in Anthropology, } \\
\text { Robert Launay, Editor } \\
\text { Anthropology and Education } \\
\text { Quarterly, Henry Trueba, Editor } \\
\text { Anthropology and Humanism } \\
\text { Quarterly, John Stewart, Editor } \\
\text { Cultural Anthropology, George } \\
\text { Marcus, Editor } \\
\text { City \& Society, Constance P. } \\
\text { de Roche, Editor } \\
\text { Medical Anthropology Quarterly, } \\
\text { Alan Harwood, Editor } \\
\text { Ethos, Robert Paul, Editor }\end{array}$ & \\
\hline
\end{tabular}

AMINTAPHIL (American Section of the International Association for the Philosophy of Law and Social Philosophy) c/o Robert C. L. Moffat, Amintaphil, School of Law, University of Florida, Gainesville, FL 32611

Phone: (904) 392-2211

Bitnet: Moffat@NERVM
Executive Director: Robert C. L. Moffat

President: Rex Martin
Public Opinion Quarterly AAPOR Newsletter
May 18-21, 1995

Bonaventure Hotel

Ft. Lauderdale, FL

P.O. Box 1248

Ann Arbor, MI 48106

Phone: (313) 764-1555 


\begin{tabular}{|c|c|c|}
\hline Organization & Officers & Publications \\
\hline $\begin{array}{l}\text { AMERICAN ASSOCIATION } \\
\text { OF POLITICAL } \\
\text { CONSULTANTS } \\
5335 \text { Wisconsin Ave., NW, } \\
\text { Ste. } 700 \text {, Washington, DC } \\
20015 \\
\text { Phone: (202) 686-5908 } \\
\text { Fax: (202) 686-7080 }\end{array}$ & $\begin{array}{l}\text { President: Thomas Edmonds } \\
\text { Executive Director: Deborah S. Malone }\end{array}$ & $\begin{array}{l}\text { Politea (Newsletter) } \\
\text { Membership Directory in the Political } \\
\quad \text { Resource Directory }\end{array}$ \\
\hline $\begin{array}{l}\text { *AMERICAN } \\
\text { CONSORTIUM FOR } \\
\text { INTERNATIONAL PUBLIC } \\
\text { ADMINISTRATION (ACIPA) } \\
\text { 1120 G Street, NW, Suite 730, } \\
\text { Washington, DC } 20005 \\
\text { Phone: }(202) 628-8965 \\
\text { Fax: }(202) 626-4978\end{array}$ & $\begin{array}{l}\text { President: Dona Wolf } \\
\text { Executive Director: vacant }\end{array}$ & $\begin{array}{l}\text { Global Network (quarterly } \\
\text { newsletter), Jamie Langlie, Editor }\end{array}$ \\
\hline $\begin{array}{l}\text { AMERICAN } \\
\text { CORRECTIONAL } \\
\text { ASSOCIATION (ACA) } \\
\text { 8025 Laurel Lakes Court, } \\
\text { Laurel, MD 20707-5075 } \\
\text { Phone: (301) 206-5100 } \\
\text { Fax: (301) 206-5061 }\end{array}$ & Executive Director: James A. Gondles, Jr. & $\begin{array}{l}\text { "On the Line"' (bi-monthly } \\
\text { newsletter), Kurt S. Olsson, } \\
\text { Managing Editor } \\
\text { Corrections Today (bi-monthly } \\
\text { magazine), Kurt S. Olsson, } \\
\text { Managing Editor } \\
\text { Juvenile and Adult Correctional } \\
\text { Departments, Institutions, } \\
\text { Agencies and Paroling Authorities } \\
\text { (of the United States and Canada) } \\
\text { (annual directory) } \\
160 \text { other publications }\end{array}$ \\
\hline
\end{tabular}

Meetings

Annual conference and regional ones
AMERICAN ECONOMIC Secretary-Treasurer: C. Elton Hinshaw ASSOCIATION

2014 Broadway, Suite 305,

Nashville, TN 37203

Phone: (615) 322-2595

Fax: (615) 343-7590
American Economic Review, Orley Ashenfelter, Editor

Journal of Economic Literature, John Pencavel, Editor

Joumal of Economic Perspectives, Carl Shapiro, Editor

AMERICAN HISTORICAL President: John H. Coatsworth, ASSOCIATION

400 A Street, SE,

Washington, DC 20003

Phone: (202) 544-2422

Fax: (202) 544-8307
Harvard University

President-elect: Vacant

Immediate Past President: Thomas C. Holt, University of Chicago

Acting Executive Director: James B. Gardner
American Historical Review,

David L. Ransel, Editor

Perspectives (newsletter)

Doctoral Dissertations in History, Directory of History Departments and Organizations, Grants and

Fellowships of Interest to Historians, Annual Report, Directory of Affiliated Societies, History Pamphlet Series, Guide to Historical Literature (3rd edition)

AMERICAN

PSYCHOLOGICAL

ASSOCIATION

750 First Street, NE,

Washington, DC 20002-4242
Chief Executive Officer: Raymond D.

Fowler

President: Ronald E. Fox
American Psychologist

30 other periodicals
Bimonthly Board

Meetings (ACIPA is

U.S. National Section of the International Institute of

Administrative

Sciences)

Annual IIAS Meetings:

1995: Riyadh, Saudi

Arabia

1996: Beijing, China

Winter Conference

January of each year

1995: Dallas, TX

1996: Philadelphia, PA

Annual Congress of

Correction

August of each year

1995: Cincinnati, $\mathrm{OH}$

1996: Nashville, TN
AMERICAN SOCIETY FOR President: Michael Walzer, IAS

POLITICAL AND LEGAL Secretary: K. I. Winston, Wheaton College

PHILOSOPHY

c/o K. I. Winston, Knapton

Hall, Wheaton College,

Norton, MA 02766

Phone: (508) 285-7722
NOMOS, Ian Shapiro, Editor
January 6-8, 1995

Washington, DC

January 5-7, 1996

San Francisco, CA 


\begin{tabular}{|c|c|c|c|}
\hline Organization & Officers & Publications & Meetings \\
\hline $\begin{array}{l}\text { *AMERICAN SOCIETY } \\
\text { FOR PUBLIC } \\
\text { ADMINISTRATION } \\
\text { 1120 G Street, NW, Suite } \\
\text { 700, Washington, DC } 20005 \\
\text { Phone: (202) 393-7878 } \\
\text { Fax: (202) 638-4952 }\end{array}$ & $\begin{array}{l}\text { President: Christine Gibbs } \\
\text { Executive Director: John P. Thomas }\end{array}$ & $\begin{array}{l}\text { Public Administration Review, David } \\
\text { H. Rosenbloom, Editor-in-Chief } \\
\text { P.A. Times, John P. Thomas, } \\
\text { Editor-in-Chief }\end{array}$ & $\begin{array}{l}\text { National conference } \\
\text { Policy conference } \\
\text { Special focus } \\
\text { conferences } \\
\text { Regional conferences }\end{array}$ \\
\hline $\begin{array}{l}\text { AMERICAN SOCIETY OF } \\
\text { INTERNATIONAL LAW } \\
2223 \text { Massachusetts Avenue, } \\
\text { NW, Washington, DC } 20008 \\
\text { Phone: (202) } 939-6000 \\
\text { Fax: (202) } 797-7133\end{array}$ & $\begin{array}{l}\text { President: Edith Brown Weiss } \\
\text { Executive Director: Charlotte Ku }\end{array}$ & $\begin{array}{l}\text { International Legal Materials, } \\
\text { Marilou M. Righini, Editor } \\
\text { American Journal of International } \\
\text { Law, Theodor Meron and } \\
\text { Petlev Vagts, Co-Editors-in-Chief } \\
\text { Proceedings, J. Laawrence Hargrove, } \\
\text { Editor } \\
\text { Occasional Papers }\end{array}$ & $\begin{array}{l}\text { Annual Meetings: } \\
\text { April 5-8, 1995 } \\
\text { Washington, DC } \\
\text { March 27-30, 1996 }\end{array}$ \\
\hline $\begin{array}{l}\text { *AMERICAN } \\
\text { SOCIOLOGICAL } \\
\text { ASSOCIATION } \\
1722 \text { N Street, NW, } \\
\text { Washington, DC } 20036 \\
\text { Phone: (202) 833-3410 } \\
\text { Fax: (202) } 785-0146\end{array}$ & $\begin{array}{l}\text { Executive Officer: Felice J. Levine } \\
\text { President: Seymour Martin Lipset, } \\
\text { George Mason University }\end{array}$ & $\begin{array}{l}\text { American Sociological Review, } \\
\text { Gerald Marwell, Editor } \\
\text { Contemporary Sociology, Walter W. } \\
\text { Powell, Editor }\end{array}$ & \\
\hline $\begin{array}{l}\text { *AMERICAN } \\
\text { STATISTICAL } \\
\text { ASSOCIATION } \\
\text { 1429 Duke Street, Alexandria, } \\
\text { VA 22314-3402 } \\
\text { Phone: (703) 684-1221 } \\
\text { Fax: (703) 684-2037 }\end{array}$ & $\begin{array}{l}\text { President: J. Stuart Hunter } \\
\text { President-Elect: Ronald L. Iman } \\
\text { Executive Director: Barbara A. Bailar }\end{array}$ & $\begin{array}{l}\text { Journal of the American Statistical } \\
\text { Association } \\
\text { The American Statistician } \\
\text { Amstat News } \\
\text { Technometrics } \\
\text { Journal of Educational Statistics } \\
\text { Current Index to Statistics } \\
\text { Journal of Business \& Economic } \\
\text { Statistics } \\
\text { Stats } \\
\text { Joumal of Computational and } \\
\text { Graphical Statistics } \\
\text { Chance }\end{array}$ & $\begin{array}{l}\text { National Conference } \\
\text { 1995: Orlando, FL } \\
\text { 1996: Chicago, IL }\end{array}$ \\
\hline $\begin{array}{l}\text { ASHBROOK CENTER FOR } \\
\text { PUBLIC AFFAIRS } \\
\text { Ashland University } \\
\text { Ashland, OH } 44805 \\
\text { Phone: (419) } 289-5411 \\
\text { Fax: (419) } 289-5425\end{array}$ & $\begin{array}{l}\text { Director: Charles Parton } \\
\text { Deputy Director: Bradford P. Wilson } \\
\text { Associate Director: Peter W. Schramm } \\
\text { Coordinator for Publications and Special } \\
\text { Programs: C. Bradley Thompson } \\
\text { Senior Fellow: Ken Masugi }\end{array}$ & $\begin{array}{l}\text { On Principle } \\
\text { The Ashbrook Principle } \\
\text { Res Publica } \\
\text { Ashbrook Essays } \\
\text { Scholarly Books }\end{array}$ & $\begin{array}{l}\text { The John M. Ashbrook } \\
\text { Memorial Dinner } \\
\text { The Ashbrook Major } \\
\text { Issues Lecture Series } \\
\text { Institute for American } \\
\text { Constitutional } \\
\text { Government }\end{array}$ \\
\hline $\begin{array}{l}\text { *ASSOCIATION FOR THE } \\
\text { ADVANCEMENT OF } \\
\text { POLICY, RESEARCH AND } \\
\text { DEVELOPMENT IN THE } \\
\text { THIRD WORLD } \\
\text { P.O. Box 70257, Washington, } \\
\text { DC 20024-0257 } \\
\text { Phone: (202) 723-7010 } \\
\text { Fax: (202) } 723-7010\end{array}$ & $\begin{array}{l}\text { Executive Director: Mekki Mtewa, } \\
\text { International Development Foundation } \\
\text { President: Larissa Grunig } \\
\text { Vice President: Ghada Talhami } \\
\text { Secretary: Elizabeth Brooks } \\
\text { Treasurer: Ritchard M'Bayo }\end{array}$ & $\begin{array}{l}\text { Science, Technology and } \\
\text { Development (book) } \\
\text { Perspectives in International } \\
\text { Development (book) } \\
\text { Program Announcements } \\
\text { International Development and } \\
\text { Alternative Futures (book) } \\
\text { International Science and Technology } \\
\text { (book) } \\
\text { Joumal of Inter-Regional Cooperation } \\
\text { and Development } \\
\text { NEWS }\end{array}$ & $\begin{array}{l}\text { Annual and Regional } \\
\text { Annual: November } \\
\text { Regional: Summer } \\
\text { Colloquia: Quarterly } \\
\text { November 1995: } \\
\text { Malawi, Africa }\end{array}$ \\
\hline
\end{tabular}




\begin{tabular}{|c|c|c|c|}
\hline Organization & Officers & Publications & Meetings \\
\hline $\begin{array}{l}\text { ASSOCIATION OF } \\
\text { AMERICAN } \\
\text { GEOGRAPHERS } \\
1710 \text { 16th Street, NW, } \\
\text { Washington, DC 20009-3198 } \\
\text { Phone: (202) 234-1450 } \\
\text { Fax: (202) 234-2744 } \\
\text { E-mail: aag@gwuvm. } \\
\text { gwu.edu or aag@gwuvm }\end{array}$ & $\begin{array}{l}\text { President: Stephen S. Birdsall } \\
\text { Vice President: Judy Olson } \\
\text { Secretary: Janet E. Kodras } \\
\text { Executive Director: Ronald F. Abler }\end{array}$ & & $\begin{array}{l}\text { March 14-18, } 1995 \\
\text { Chicago, IL }\end{array}$ \\
\hline $\begin{array}{l}\text { *ASSOCIATION FOR } \\
\text { COMPARATIVE } \\
\text { ECONOMIC STUDIES } \\
\text { Michael R. Dohan, Dept. of } \\
\text { Economics, Queens College/ } \\
\text { CUNY, Flushing, NY } 11367 \\
\text { Phone: (718) } 997-5461 \\
\text { Fax: (516) 549-2032 }\end{array}$ & $\begin{array}{l}\text { President: Elizabeth Clayton (1991); } \\
\text { Zbigniew M. Fallenbuchl (1992) } \\
\text { President-elect 1993: Holland Hunter } \\
\text { Executive Secretary: Michael R. Dohan }\end{array}$ & $\begin{array}{l}\text { Comparative Economic Studies } \\
\text { Journal of Comparative Economics }\end{array}$ & \\
\hline $\begin{array}{l}\text { *ASSOCIATION OF } \\
\text { KOREAN POLITICAL } \\
\text { SCIENTISTS IN NORTH } \\
\text { AMERICA } \\
\text { 1021 St. Clair Street } \\
\text { Hagerstown, MD } 21740\end{array}$ & $\begin{array}{l}\text { President: Hang Yul Rhee } \\
\text { Secretary-General: Jong Oh Ra }\end{array}$ & $\begin{array}{l}\text { Political Studies Review, Hang Yul } \\
\text { Rhee, Editor } \\
\text { AKPSNA Newsletter, Jong Oh Ra, } \\
\text { Editor }\end{array}$ & $\begin{array}{l}\text { Annual meeting in } \\
\text { conjunction with APSA } \\
\text { annual convention or } \\
\text { Association of Asian } \\
\text { Studies annual meeting }\end{array}$ \\
\hline $\begin{array}{l}\text { *ASSOCIATION OF } \\
\text { PUBLIC DATA USERS } \\
\text { Princeton University } \\
\text { Computing Center, } 87 \\
\text { Prospect Ave., } \\
\text { Princeton, NJ } 08544-1002 \\
\text { Phone: (609) 258-6025 }\end{array}$ & $\begin{array}{l}\text { President: Janie Harris } \\
\text { Vice President: Joseph Salvo } \\
\text { Executive Director: Susan Anderson }\end{array}$ & APDU Newsletter (monthly) & \\
\hline $\begin{array}{l}\text { ASSOCIATION FOR } \\
\text { PUBLIC POLICY } \\
\text { ANALYSIS AND } \\
\text { MANAGEMENT } \\
\text { P.O. Box 18766, Washington, } \\
\text { DC 20036-8766 } \\
\text { Phone: (202) } 857-8788 \\
\text { Fax: (202) 466-3982 } \\
\text { E-mail: appam@vi.urban.org }\end{array}$ & $\begin{array}{l}\text { President: Ellen Schall, New York } \\
\text { University } \\
\text { Executive Director: Dante Noto }\end{array}$ & $\begin{array}{l}\text { Journal of Policy Analysis and } \\
\text { Management, Janet Rothenberg } \\
\text { Pack, Editor }\end{array}$ & $\begin{array}{l}\text { Annual research } \\
\text { conference } \\
\text { November } 2-4,1995: \\
\text { Washington, DC }\end{array}$ \\
\hline $\begin{array}{l}\text { *ASSOCIATION FOR } \\
\text { POLITICS AND THE } \\
\text { LIFE SCIENCES } \\
\text { Northern Illinois University, } \\
\text { DeKalb, IL } 60115-2854 \\
\text { Phone: (815) } 753-9675 \\
\text { Fax: (815) 753-2305 }\end{array}$ & $\begin{array}{l}\text { Chairperson of the Council: Joseph Losco } \\
\text { Vice-Chairperson of the Council: } \\
\text { Denise Baer } \\
\text { Recording Secretary of the Council: } \\
\text { Steven Peterson } \\
\text { Treasurer of the Council: James N. Schubert } \\
\text { Executive Director: James N. Schubert }\end{array}$ & $\begin{array}{l}\text { Politics and the Life Sciences, } \\
\text { Gary R. Johnson, Lake Superior } \\
\text { State University, Editor; Andrea L. } \\
\text { Bonnicksen, Northern Illinois } \\
\text { University, and Joseph Losco, Ball } \\
\text { State University, Book Review } \\
\text { Editors: Beech Tree Publishing, } \\
\text { United Kingdom, Publisher }\end{array}$ & $\begin{array}{l}\text { In conjunction with } \\
\text { APSA annual meeting }\end{array}$ \\
\hline $\begin{array}{l}\text { *THE CENTER FOR THE } \\
\text { STUDY OF THE } \\
\text { CONSTITUTION } \\
\text { P.O. Box } 897 \text {, Carlisle, PA } \\
17013\end{array}$ & $\begin{array}{l}\text { Executive Director: Eugene W. Hickok, Jr., } \\
\text { Dickinson College } \\
\text { Director: Gary L. McDowell } \\
\text { Director of Special Programs: Jeffrey Leigh } \\
\text { Sedgwick, University of Massachusetts }\end{array}$ & $\begin{array}{c}\text { Benchmark, Eugene Hickok, } \\
\text { Dickinson College, Editor }\end{array}$ & $\begin{array}{l}\text { In conjunction with the } \\
\text { APSA annual meeting }\end{array}$ \\
\hline $\begin{array}{l}\text { CENTER FOR THE STUDY } \\
\text { OF FEDERALISM } \\
\text { Temple University, } 300-00 \\
1616 \text { Walnut Street, } \\
\text { Room 507, } \\
\text { Philadelphia, PA } 19103 \\
\text { Phone: (215) 204-1480 } \\
\text { Fax: (215) 204-7784 }\end{array}$ & $\begin{array}{l}\text { Director: Daniel J. Elazar } \\
\text { Project Coordinator: } \\
\text { Fellows: Ellis Katz, Temple University; } \\
\text { John Kincaid, Lafayette College; Donald } \\
\text { Lutz, University of Houston; Stephen } \\
\text { Schechter, Russell Sage College; G. Alan } \\
\text { Tarr, Rutgers University }\end{array}$ & $\begin{array}{l}\text { PUBLIUS: The Journal of Federalism } \\
\text { Covenant Letter } \\
\text { The Federalism Report }\end{array}$ & $\begin{array}{l}\text { Conference for Federal } \\
\text { Studies, in conjunction } \\
\text { with the APSA annual } \\
\text { meeting }\end{array}$ \\
\hline
\end{tabular}




\begin{tabular}{|c|c|c|c|}
\hline Organization & Officers & Publications & Meetings \\
\hline $\begin{array}{l}{ }^{*} \text { CENTER FOR THE } \\
\text { STUDY OF JUDICIAL } \\
\text { POWER } \\
\text { Program on Courts and Public } \\
\text { Policy, McGraw Hall, Cornell } \\
\text { University, Ithaca, NY } 14853\end{array}$ & $\begin{array}{l}\text { Co-Directors: Jeremy Rabkin, } \\
\text { William Kristol }\end{array}$ & Occasional & $\begin{array}{l}\text { In conjunction with the } \\
\text { APSA annual meeting }\end{array}$ \\
\hline $\begin{array}{l}\text { CITIZENS' RESEARCH } \\
\text { FOUNDATION } \\
\text { University of Southern } \\
\text { California, Research Annex, } \\
3716 \text { S. Hope Street, Los } \\
\text { Angeles, CA } 90007 \\
\text { Phone: (213) 743-5211 } \\
\text { Fax: (213) 743-3130 }\end{array}$ & Director: Herbert E. Alexander & Occasional & Occasional \\
\hline $\begin{array}{l}\text { THE CLAREMONT } \\
\text { INSTITUTE FOR } \\
\text { THE STUDY OF } \\
\text { STATESMANSHIP AND } \\
\text { POLITICAL PHILOSOPHY } \\
\text { The Claremont Institute, 250 } \\
\text { W. First Street, Suite } 330 \text {, } \\
\text { Claremont, CA } 91711 \\
\text { Phone: (909) 621-6825 } \\
\text { Fax: (909) 626-8724 }\end{array}$ & $\begin{array}{l}\text { President: L. P. Arnn } \\
\text { Vice President: Charles Heatherly } \\
\text { Fellows: William R. Rusher; Harry V. Jaffa; } \\
\text { Charles R. Kesler, Claremont McKenna } \\
\text { College; Thomas West, University of } \\
\text { Dallas; John Marini, University of } \\
\text { Nevada-Reno; Ken Masugi, Hillsdale; } \\
\text { Christopher Flannery, Azusa Pacific } \\
\text { University }\end{array}$ & $\begin{array}{l}\text { National Review West } \\
\text { The Public View Briefings }\end{array}$ & $\begin{array}{l}\text { Panels at APSA annual } \\
\text { meetings; occasional } \\
\text { conferences }\end{array}$ \\
\hline $\begin{array}{l}\text { COMMITTEE FOR } \\
\text { PARTY RENEWAL } \\
\text { John White, Department of } \\
\text { Political Science, Catholic } \\
\text { University, Washington, DC } \\
20064 \\
\text { Phone: (202) } 319-5128\end{array}$ & $\begin{array}{l}\text { Co-Chairmen: Cong. Bill Thomas, } \\
\text { John White } \\
\text { Executive Director: Paul Hernson } \\
\text { Editor: Candice Nelson }\end{array}$ & Party Line (bi-monthly newsletter) & $\begin{array}{l}\text { Annual business } \\
\text { meetings and panels at } \\
\text { APSA convention }\end{array}$ \\
\hline $\begin{array}{l}\text { COMMITTEE ON } \\
\text { HEALTH POLITICS } \\
\text { c/o Jim Brasfield, Webster } \\
\text { University, } 470 \text { East } \\
\text { Lockwood, St. Louis, MO } \\
\text { 73119-3194 } \\
\text { Phone: (314) 968-7063 } \\
\text { Fax: (314) 968-7077 }\end{array}$ & & $\begin{array}{l}\text { Journal of Health Politics, Policy } \\
\text { and Law, Mark Peterson, Editor }\end{array}$ & $\begin{array}{l}\text { At the APSA annual } \\
\text { meeting and with the } \\
\text { American Public Health } \\
\text { Association }\end{array}$ \\
\hline $\begin{array}{l}\text { COMMUNAL STUDIES } \\
\text { ASSOCIATION } \\
\text { c/o Donald E. Pitzer, Center } \\
\text { for Communal Studies, } \\
\text { University of Southern } \\
\text { Indiana, Evansville, IN } 47712 \\
\text { Phone: (812) 464-1727 } \\
\text { Fax: (812) } 464-1960\end{array}$ & $\begin{array}{l}\text { President: Lanny Haldy } \\
\text { Vice President: Harvey Barker } \\
\text { Executive Secretary: Donald Janzen } \\
\text { Treasurer: Gina Walker }\end{array}$ & $\begin{array}{l}\text { Communal Societies (annual) } \\
\text { Communal Studies Association } \\
\text { Newsletter (twice yearly) }\end{array}$ & $\begin{array}{l}\text { Annual Meeting } \\
\text { Oct. 12-14, } 1995 \\
\text { Estero, FL }\end{array}$ \\
\hline $\begin{array}{l}{ }^{*} \text { CONFERENCE FOR THE } \\
\text { STUDY OF POLITICAL } \\
\text { THOUGHT } \\
\text { Sharon Nickel Showiss, Dept. } \\
\text { of Political Science, Pitzer } \\
\text { College, Claremont, CA } \\
91711 \\
\text { Phone: (714) 621-8218 } \\
\text { Fax: (714) 621-8481 }\end{array}$ & $\begin{array}{l}\text { Chair: Alan Ryan, Princeton University } \\
\text { Secretary-Treasurer: Sharon Nickel } \\
\text { Snowiss, Pitzer College }\end{array}$ & $\begin{array}{l}\text { Conference for the Study of Political } \\
\text { Thought (newsletter), Simone } \\
\text { Chambers, University of Colorado, } \\
\text { Boulder, Editor }\end{array}$ & Annual meeting \\
\hline
\end{tabular}




\begin{tabular}{|c|c|c|c|}
\hline Organization & Officers & Publications & Meetings \\
\hline $\begin{array}{l}\text { *CONFERENCE GROUP } \\
\text { ON JURISPRUDENCE } \\
\text { AND PUBLIC LAW } \\
\text { c/o Karol Soltan, Dept. of } \\
\text { Government and Politics, } \\
\text { University of Maryland, } \\
\text { College Park, MD 0742 } \\
\text { Fax: (301) 314-9690 } \\
\text { E-mail: } \\
\text { ksoltan@bss2.umd.edu }\end{array}$ & Coordinators: & & $\begin{array}{l}\text { In conjunction with } \\
\text { APSA annual meeting }\end{array}$ \\
\hline $\begin{array}{l}\text { CONFERENCE GROUP } \\
\text { ON POLICY STUDIES } \\
\text { AND PUBLIC LAW } \\
\text { c/o Ira H. Carmen, Dept. of } \\
\text { Political Science, University } \\
\text { of Illinois, Urbana, IL } 61801 \\
\text { Phone: (217) 333-3881 } \\
\text { Fax: (217) 244-5712 }\end{array}$ & Program Organizer: Ira H. Carmen & & $\begin{array}{l}\text { Panels conducted at } \\
\text { APSA annual meeting }\end{array}$ \\
\hline $\begin{array}{l}\text { ERIC VOEGELIN SOCIETY } \\
\text { c/or Eric Voegelin Institute, } \\
\text { Louisiana State University, } \\
\text { 240 Stubbs Hall, Baton } \\
\text { Rouge, LA 70803-5466 } \\
\text { Phone: (504) 388-2552/-3288 } \\
\text { Fax: (504) 388-2540 }\end{array}$ & Secretary: Ellis Sandoz & Newsletter (annual, July 15) & $\begin{array}{l}\text { In conjunction with } \\
\text { APSA annual meeting }\end{array}$ \\
\hline $\begin{array}{l}\text { FOREIGN POLICY } \\
\text { RESEARCH INSTITUTE } \\
\text { 3615 Chestnut Street, } \\
\text { Philadelphia, PA } 19104 \\
\text { Phone: (215) 382-0685 } \\
\text { Fax: (215) 382-0131 }\end{array}$ & $\begin{array}{l}\text { President: Harvey Sicherman } \\
\text { Vice President: Alan Luxenberg }\end{array}$ & $\begin{array}{l}\text { Orbis } \\
\text { Peacefacts } \\
\text { FPRI Wire } \\
\text { Middle East Council Wire } \\
\text { Back Channel: FPRI Newsletter }\end{array}$ & Occasional \\
\hline $\begin{array}{l}{ }^{*} \text { GAY AND LESBIAN } \\
\text { CAUCUS FOR POLITICAL } \\
\text { SCIENCE } \\
\text { c/o Mark Blasius, City } \\
\text { University of New York, } 429 \\
\text { W. 46th St., Apt. 6-D, New } \\
\text { York, NY 10036, (212) 246- } \\
\text { 1256; or Sarah Slavin, Buffalo } \\
\text { State College, Political } \\
\text { Science Dept., 1300 Elmwood } \\
\text { Ave., Buffalo, NY 14222, } \\
\text { (716) 878-6205 }\end{array}$ & $\begin{array}{l}\text { Co-Chairs: Mark Blasius, Sarah Slavin } \\
\text { Secretary-Treasurer: Jackie Stevens, } \\
\text { University of California-Berkeley } \\
\text { Program Organizer: Kenneth Sherrill, } \\
\text { Hunter College/CUNY }\end{array}$ & $\begin{array}{l}\text { Newsletter of the Gay and Lesbian } \\
\text { Caucus for Political Science, } \\
\text { Robert Bailey, Columbia } \\
\text { University, Co-editor }\end{array}$ & $\begin{array}{l}\text { At the APSA annual } \\
\text { meetings }\end{array}$ \\
\hline $\begin{array}{l}\text { *GAY, LESBIAN, } \\
\text { BISEXUAL POLITICAL } \\
\text { SCIENCE CAUCUS } \\
\text { c/o Joan C. Tronto, Women's } \\
\text { Studies Program, Hunter } \\
\text { College, } 695 \text { Park Avenue, } \\
\text { New York, NY } 10024 \\
\text { Phone: }(212) 772-5681 \\
\text { Fax: (212) 650-3669 } \\
\text { E-mail: } \\
\text { jcthc@cunyvm.cuny.edu }\end{array}$ & $\begin{array}{l}\text { Chair: Joan C. Tronto } \\
\text { Chair-Elect and Program Chair: } \\
\text { Gregory Lewis } \\
\text { Secretary/Treasurer: Leonard Hirsch }\end{array}$ & $\begin{array}{l}\text { Newsletter of the Gay, Lesbian, } \\
\text { Bisexual Political Science Caucus, } \\
\text { Leonard Hirsch, Editor }\end{array}$ & $\begin{array}{l}\text { At the APSA Annual } \\
\text { Meeting }\end{array}$ \\
\hline $\begin{array}{l}\text { GUATEMALA SCHOLARS } \\
\text { NETWORK } \\
\text { Robert H. Trudeau, Political } \\
\text { Science Dept., Providence } \\
\text { College, Providence, RI } 02918 \\
\text { Phone: (401) 865-2434 } \\
\text { E-mail: NP180039@brown.vm }\end{array}$ & $\begin{array}{l}\text { Coordinator: Marilyn Moors, Route 1, } \\
\text { Box 55, Friendsville, MD } 21531 \\
\text { Political Science Discipline Chairperson: } \\
\text { Robert H. Trudeau }\end{array}$ & & $\begin{array}{l}\text { In conjunction with the } \\
\text { APSA annual meeting } \\
\text { and LASA Congress }\end{array}$ \\
\hline
\end{tabular}




\begin{tabular}{|c|c|c|c|}
\hline Organization & Officers & Publications & Meetings \\
\hline $\begin{array}{l}\text { INDIAN POLICY } \\
\text { NETWORK } \\
\text { Barry University, } 11300 \text { N.E. } \\
\text { 2nd Ave., Miami, FL } 33161 \\
\text { Phone: (307) 268-2713 } \\
\text { Fax: (307) 268-2416 }\end{array}$ & $\begin{array}{l}\text { Coordinator: Margaret M. Murdock, } \\
\text { University of Wyoming, Casper }\end{array}$ & $\therefore$ & $\begin{array}{l}\text { In conjunction with the } \\
\text { APSA annual meeting } \\
\text { and the Western Social } \\
\text { Science }\end{array}$ \\
\hline $\begin{array}{l}\text { INTERNATIONAL } \\
\text { COMMUNAL STUDIES } \\
\text { ASSOCIATION } \\
\text { c/o Donald E. Pitzer, Center } \\
\text { for Communal Studies, } \\
\text { University of Southern } \\
\text { Indiana, Evansville, IN } 47712\end{array}$ & $\begin{array}{l}\text { Executive Director: Yaacov Oved } \\
\text { President: Pearl W. Bartelt } \\
\text { Vice President: Dennis Hardy }\end{array}$ & $\begin{array}{l}\text { Bulletin } \\
\text { (three times yearly) }\end{array}$ & $\begin{array}{l}\text { Triennial International } \\
\text { Conference } \\
\text { May } 1995 \text { Israel }\end{array}$ \\
\hline $\begin{array}{l}\text { INTERNATIONAL } \\
\text { FEDERATION OF } \\
\text { VEXILLOLOGICAL } \\
\text { ASSOCIATIONS (study of } \\
\text { political symbols, e.g., flags) } \\
\text { Flag Research Center, Box } \\
\text { 580, Winchester, MA } 01890 \\
\text { Phone: (617) 729-9410 } \\
\text { Fax: (617) } 721-4817\end{array}$ & Secretary-General: Whitney Smith & $\begin{array}{l}\text { The Flag Bulletin (bi-monthly) } \\
\text { Info FIAV (biannual) } \\
\text { Proceedings (biennial) }\end{array}$ & $\begin{array}{l}\text { Annual Meeting } \\
\text { 1995: Warsaw }\end{array}$ \\
\hline $\begin{array}{l}\text { INTERNATIONAL PEACE } \\
\text { RESEARCH INSTITUTE, } \\
\text { OSLO } \\
\text { Fuglehauggata } 11 \text {, } \\
0260 \text { Oslo } 2 \text {, Norway } \\
\text { Phone: }(+47) 22557150 \\
\text { Fax: }(+47) 22558422\end{array}$ & $\begin{array}{l}\text { Director: Dan Smith } \\
\text { Information Director: Ole Berthelsen }\end{array}$ & $\begin{array}{l}\text { Security Dialogue } \\
\text { Jourmal of Peace Research } \\
\quad \text { (quarterlies) }\end{array}$ & \\
\hline $\begin{array}{l}\text { INTERNATIONAL } \\
\text { PERSONNEL } \\
\text { MANAGEMENT } \\
\text { ASSOCIATION } \\
\text { 1617 Duke Street, } \\
\text { Alexandria, VA } 22314 \\
\text { Phone: (703) 549-7100 } \\
\text { Fax: (703) 684-0948 }\end{array}$ & Executive Director: Donale K. Tichenor & $\begin{array}{l}\text { Public Personnel Management } \\
\text { IPMA News } \\
\text { Agency Issues }\end{array}$ & $\begin{array}{l}\text { Annual International } \\
\text { Training Conference } \\
\text { 1995: Baltimore, MD } \\
\text { 1996: Las Vegas, NJ }\end{array}$ \\
\hline $\begin{array}{l}\text { INTERNATIONAL } \\
\text { POLITICAL SCIENCE } \\
\text { ASSOCIATION } \\
\text { c/o Department of Politics } \\
\text { University College, Dublin } \\
\text { Belfield, Dublin 4 Ireland } \\
\text { Phone: + } 353-1-2693244 \\
\text { Fax: }+353-1-7061171\end{array}$ & $\begin{array}{l}\text { President: Jean Leca } \\
\text { Secretary-General: John Coakley } \\
\text { Administrator: Louise Delaney }\end{array}$ & $\begin{array}{l}\text { Participation (newsletter), } \\
\text { John Coakley and Louise Delaney, } \\
\text { Co-editors } \\
\text { International Political Science } \\
\text { Review, John Meisel, Nazli Choucri } \\
\text { and Jean Laponce, Editors } \\
\text { International Political Science } \\
\text { Abstracts, Serge Hurtig, Editor } \\
\text { Advances in Political Science: An } \\
\text { Internatinal Series, Asher Arian, } \\
\text { Editor }\end{array}$ & $\begin{array}{l}\text { 1998: Seoul, South } \\
\text { Korea }\end{array}$ \\
\hline $\begin{array}{l}\text { INTERNATIONAL } \\
\text { SOCIETY OF POLITICAL } \\
\text { PSYCHOLOGY } \\
\text { Dept. of Political Science, } \\
\text { 304 Stetson Hall, Williams } \\
\text { College, Williamstown, MA } \\
01267 \\
\text { Phone: (413) 597-2538 } \\
\text { Fax: (413) 597-4200 } \\
\text { Internet: george.e.marcus@ } \\
\text { williams.edu. }\end{array}$ & $\begin{array}{l}\text { President: David O. Sears } \\
\text { Executive Director: George E. Marcus }\end{array}$ & $\begin{array}{l}\text { Political Psychology (quarterly) } \\
\text { ISPP News (semi-annual) }\end{array}$ & $\begin{array}{l}\text { 18th Annual Scientific } \\
\text { Meeting } \\
\text { July 6-10, } 1995 \\
\text { Washington, DC }\end{array}$ \\
\hline
\end{tabular}




\begin{tabular}{|c|c|c|c|}
\hline Organization & Officers & Publications & Meetings \\
\hline $\begin{array}{l}\text { INTERNATIONAL } \\
\text { STUDIES ASSOCIATION } \\
\text { c/o W. Ladd Hollist, } \\
\text { Executive Director, David M. } \\
\text { Kennedy Center for } \\
\text { International Studies, } 216 \\
\text { HRCB, Brigham Young } \\
\text { University, Provo, UT } 84602 \\
\text { Phone: (801) 378-5459 } \\
\text { Fax: (801) 378-7075 }\end{array}$ & $\begin{array}{l}\text { President: Ted Robert Gurr, University of } \\
\text { Maryland } \\
\text { President-elect: Susan Strange, } \\
\text { University of Warwick }\end{array}$ & $\begin{array}{l}\text { International Studies Quarterly, } \\
\text { Ohio State University, Editor } \\
\text { ISA Newsletter, Brigham Young } \\
\text { University, Editor } \\
\text { IS Notes, American Graduate School } \\
\text { of International Management, } \\
\text { Editor }\end{array}$ & $\begin{array}{l}\text { 35th Annual Convention } \\
\text { 36th Annual Convention: } \\
\text { February 22-26, } 1995 \\
\text { Chicago, IL }\end{array}$ \\
\hline $\begin{array}{l}\text { INTER-UNIVERSITY } \\
\text { SEMINAR ON ARMED } \\
\text { FORCES AND SOCIETY } \\
\text { Northwestern University } \\
618 \text { Garrett Place } \\
\text { Evanston, IL } 60208 \\
\text { Phone: (708) 467-3041 } \\
\text { Fax: (708) } 467-3043\end{array}$ & $\begin{array}{l}\text { Chairman: Charles C. Moskos } \\
\text { Executive Director: John Allen Williams } \\
\text { Assistant Executive Director: } \\
\text { Robert A. Vitas } \\
\text { Secretary and Treasurer: Cynthia Watson } \\
\text { Journal Editor: James Burk } \\
\text { Newsletter Editor: Hitoshi Kawano }\end{array}$ & $\begin{array}{l}\text { Armed Forces and Society } \\
\text { Newsletter } \\
\text { IUS Special Editions on Armed } \\
\text { Forces and Society }\end{array}$ & $\begin{array}{l}\text { Biennial Internatinal } \\
\text { Conference } \\
\text { 1995: Baltimore, MD }\end{array}$ \\
\hline $\begin{array}{l}\text { LAW AND SOCIETY } \\
\text { ASSOCIATION } \\
\text { Hampshire House, Box } \\
\text { 33615, University of } \\
\text { Massachusetts, Amherst, MA } \\
\text { 01003-3615 } \\
\text { Phone: (413) 545-4617 } \\
\text { Fax: (413) 545-1640 } \\
\text { E-mail: lsa@legal.umass.edu }\end{array}$ & $\begin{array}{l}\text { President: Sally E. Merry, Wellesley College } \\
\text { Secretary: Austin Sarat, Amherst College } \\
\text { Executive Officer: Ronald M. Pipkin, } \\
\text { University of Massachusetts }\end{array}$ & $\begin{array}{l}\text { Law and Society Review, William M. } \\
\text { O'Barr, Duke University, Cultural } \\
\text { Anthropology }\end{array}$ & $\begin{array}{l}\text { June } 1-4,1995 \\
\text { Toronto, Canada }\end{array}$ \\
\hline $\begin{array}{l}\text { NATIONAL ASSOCIATION } \\
\text { OF SCHOOLS OF PUBLIC } \\
\text { AFFAIRS AND } \\
\text { ADMINISTRATION } \\
1120 \text { G St., NW, Suite } 730, \\
\text { Washington, DC } 20005 \\
\text { Phone: (202) } 628-8965 \\
\text { Fax: (202) 626-4978 }\end{array}$ & $\begin{array}{l}\text { President: H. Brinton Milward, } \\
\text { University of Arizona } \\
\text { Vice President: Davis B. Bobrow } \\
\text { Executive Director: Alfred M. Zuck }\end{array}$ & $\begin{array}{l}\text { Directory of Programs in Public } \\
\text { Affairs and Administration, } 1992 \\
\text { Report of NASPAA Member } \\
\text { Institutions } \\
\text { Biannual Newsletter } \\
\text { Accreditation documents for MPA } \\
\text { degrees }\end{array}$ & Annual Meeting \\
\hline $\begin{array}{l}\text { NATIONAL CONFERENCE } \\
\text { OF BLACK POLITICAL } \\
\text { SCIENTISTS } \\
\text { c/o William H. Boone, Clark } \\
\text { Atlanta University, } \\
\text { Department of Political } \\
\text { Science, Atlanta, GA } 30314 \\
\text { Phone: (404) } 880-8719\end{array}$ & $\begin{array}{l}\text { President: William Boone, Clark Atlanta } \\
\text { University } \\
\text { President Elect: Lenneal Henderson, } \\
\text { University of Baltimore } \\
\text { Executive Director: Lois Hollis, } \\
\text { Albany State College }\end{array}$ & $\begin{array}{l}\text { National Political Science Review } \\
\text { NCOBPS Quarterly Newsletter }\end{array}$ & $\begin{array}{l}\text { Annual Meeting } \\
\text { March 9-11, } 1995 \\
\text { Baltimore, MD }\end{array}$ \\
\hline
\end{tabular}

*NATIONAL SOCIAL Executive Director: Jerry Baydo

SCIENCE ASSOCIATION

2020 Hills Lake Dr.

El Cajon, CA 92020-1018

Phone: (619) 448-4709

NATIONAL SOCIETY FOR Executive Director: Allen Wutzdorff

EXPERIENTIAL

EDUCATION

3509 Haworth Drive, Suite

207, Raleigh, NC 27609-7229

Phone: (919) 787-3263

\section{NSEE Quarterly}

National Directory of Intemships and several papers and books that examine key issues and principles of good practice in experiential education programs
Annual Meeting

November 1995

New Orleans, LA 


\begin{tabular}{|c|c|c|c|}
\hline Organization & Oficers & Publications & Meetings \\
\hline $\begin{array}{l}\text { NORTH AMERICAN } \\
\text { SOCIETY FOR SOCIAL } \\
\text { PHILOSOPHY } \\
\text { Dept. of Political Science, } \\
\text { California Polytechnic } \\
\text { University, } 3801 \text { W. Temple } \\
\text { Ave., Pomona, CA } 91768 \\
\text { Phone: (909) 869-3886 }\end{array}$ & $\begin{array}{l}\text { Political Science Division Chair: } \\
\text { David M. Speak }\end{array}$ & $\begin{array}{l}\text { The Journal of Social Philosophy } \\
\text { (quarterly) }\end{array}$ & $\begin{array}{l}\text { In conjunction with the } \\
\text { APSA annual meeting } \\
\text { and also Annual } \\
\text { International Social } \\
\text { Philosophy conference }\end{array}$ \\
\hline $\begin{array}{l}\text { PI SIGMA ALPHA } \\
\text { 1527 New Hampshire Ave., } \\
\text { NW, Washington, DC } 20036 \\
\text { Phone: (202) 483-2512 } \\
\text { Fax: (202) 483-2657 }\end{array}$ & $\begin{array}{l}\text { President: David Magleby, Brigham Young } \\
\text { University } \\
\text { President-Elect: John Bibby, University of } \\
\text { Wisconsin-Milwaukee } \\
\text { National Director: James I. Lengle }\end{array}$ & $\begin{array}{l}\text { Pi Sigma Newsletter } \\
\text { Information Booklet }\end{array}$ & $\begin{array}{l}\text { In conjunction with the } \\
\text { APSA annual meetings }\end{array}$ \\
\hline $\begin{array}{l}\text { POLICY STUDIES } \\
\text { ORGANIZATION } \\
\text { University of Illinois, } 702 \mathrm{~S} \text {. } \\
\text { Wright, Urbana, IL } 61801 \\
\text { Phone: (217) } 359-8541 \\
\text { Fax: (217) } 244-5712\end{array}$ & $\begin{array}{l}\text { President: Walter Beach, Heldref } \\
\text { Publications } \\
\text { President-Elect: Louise White, George } \\
\text { Mason University } \\
\text { Secretary-Treasurer: Stuart Nagel, } \\
\text { University of Illinois, Urbana }\end{array}$ & $\begin{array}{l}\text { Policy Studies Joumal, Uday Desai, } \\
\text { Southern Illinois University; Mack } \\
\text { Shelley, Iowa State University, } \\
\text { Co-editors } \\
\text { Policy Studies Review, Allan } \\
\text { Rosenbaum, Florida International } \\
\text { University, Editor } \\
\text { Developmental Policy Studies } \\
\text { Newsletter-Journal } \\
\text { Policy Evaluation Newsletter-Journal } \\
\text { Policy Studies Directory } \\
\text { Political Science Utilization Directory } \\
\text { Policy Grants Directory } \\
\text { Policy Research Centers Directory } \\
\text { Policy Studies Personnel Directory } \\
\text { Policy Publishers and Associations } \\
\text { Directory } \\
\text { PSO-Lexington Books Series } \\
\text { PSO-Sage Publications Series } \\
\text { PSO-Praeger Series } \\
\text { PSO-JAI Series } \\
\text { PSO-Kennikat Series } \\
\text { PSO-Macmillan Series } \\
\text { PSO-Greenwood Series } \\
\text { PSO-Nelson Hall Series } \\
\text { JAI Multi-Volume Treatises } \\
\text { PSO-UPA Series } \\
\text { PSO-Nova Science Series }\end{array}$ & \\
\hline $\begin{array}{l}\text { COMMITTEE ON THE } \\
\text { POLITICAL ECONOMY OF } \\
\text { THE GOOD SOCIETY } \\
\text { (PEGS) } \\
\text { Dept. of Government and } \\
\text { Politics, 3140 Tydings } \\
\text { University of Maryland at } \\
\text { College Park, College Park, } \\
\text { MD 20742-8221 } \\
\text { Phone: (301) } 405-4117 \\
\text { Fax: (301) } 314-9690\end{array}$ & $\begin{array}{l}\text { Executive Board: Stephen L. Elkin, Karol } \\
\text { Soltan, and Gar Alperovitz } \\
\text { Executive Director: Eric N. Lindblom } \\
\text { Program Chairman: Nicholas S. Hathaway }\end{array}$ & $\begin{array}{l}\text { PEGS Newsletter ( } 3 \text { times a year), } \\
\text { Kathryn Doherty, University of } \\
\text { Maryland at College Park, Editor }\end{array}$ & $\begin{array}{l}\text { In conjunction with } \\
\text { APSA annual meeting }\end{array}$ \\
\hline $\begin{array}{l}\text { POLITICS AND } \\
\text { LITERATURE GROUP } \\
\text { c/o Mary Nichols, } \\
\text { Department of Political } \\
\text { Science } \\
\text { Fordham University } \\
\text { Bronx, NY } 10458\end{array}$ & & $\begin{array}{l}\text { Newsletter ( } 2 / \text { year), Tricia Robertson, } \\
\text { Fordham University }\end{array}$ & $\begin{array}{l}\text { In conjunction with } \\
\text { APSA annual meeting }\end{array}$ \\
\hline
\end{tabular}




\begin{tabular}{|c|c|c|c|}
\hline Organization & Officers & Publications & Meetings \\
\hline $\begin{array}{l}\text { POLITICS OF EDUCATION } \\
\text { ASSOCIATION } \\
\text { c/o Robert Wimpelburg } \\
\text { College of Education, } \\
\text { University of New Orleans, } \\
\text { New Orleans, LA } 70148 \\
\text { Phone: (504) 286-6719 } \\
\text { Fax: (504) 286-6065 }\end{array}$ & $\begin{array}{l}\text { President: Robert Wimpelburg, University } \\
\text { of New Orleans } \\
\text { Secretary: Frances Fowler, Miami } \\
\text { University of Ohio } \\
\text { Treasurer: Louise Adler, California State } \\
\text { University-Fullerton }\end{array}$ & $\begin{array}{l}\text { Politics of Education Bulletin, } \\
\text { Carolyn Herrington, Florida State } \\
\text { University, Editor }\end{array}$ & \\
\hline $\begin{array}{l}\text { *PROGRESSIVE POLITICS } \\
\text { c/o Jim Hutter, Dept. of } \\
\text { Political Science, Iowa State } \\
\text { University, Ames, IA } 50011\end{array}$ & $\begin{array}{l}\text { President: Jim Hutter } \\
\text { Vice-President for Publications: Lois } \\
\text { Lovelace Duke, Dept. of Political Science, } \\
\text { 230-D Brackett Hall, Clemson } \\
\text { University, Clemson, SC 29634-1509 } \\
\text { Co-Founder: James MacGregor Burns, } 604 \\
\text { Bee Hill Road, Williamstown, MA } 01267\end{array}$ & & $\begin{array}{l}\text { In conjunction with the } \\
\text { APSA annual meeting } \\
\text { and the Midwest } \\
\text { Political Science } \\
\text { Association meeting }\end{array}$ \\
\hline $\begin{array}{l}\text { THE PUBLIC CHOICE } \\
\text { SOCIETY } \\
\text { c/o Charles Rowley, Center } \\
\text { for Study of Public Choice, } \\
\text { George Mason University, } \\
\text { Fairfax, VA } 22030 \\
\text { Phone: (703) } 934-6960 \\
\text { Fax: (703) 352-9747 }\end{array}$ & $\begin{array}{l}\text { President: Robert D. Tollison, Center for } \\
\text { Study of Public Choice, George Mason } \\
\text { University }\end{array}$ & $\begin{array}{l}\text { Public Choice (8 issues per annum- } \\
\text { new format effective Vol. } 71(3), \\
\text { September 1991), Charles K. } \\
\text { Rowley and Robert D. Tollison, } \\
\text { George Mason University, Editors }\end{array}$ & $\begin{array}{l}\text { Long Beach, CA } \\
\text { March 24-26, } 1995\end{array}$ \\
\hline $\begin{array}{l}\text { RESEARCH COMMITTEE } \\
\text { ON POLITICAL FINANCE } \\
\text { AND POLITICAL } \\
\text { CORRUPTION } \\
\text { c/o Institute of Comparative } \\
\text { Politics, Carl von Ossietzky } \\
\text { University, PO Box } 2503 \\
\text { D-26111 Oldenburg, Germany } \\
\text { Phone: } 49-441 / 798-2170 \\
\text { Fax: } 49-441 / 5040842\end{array}$ & $\begin{array}{l}\text { Chairman: Karl-Heinz Nassmacher, } \\
\text { Carl von Ossietzky University } \\
\text { Vice Chairman: Michael Pinto-Duschinsky, } \\
\text { Brunel University } \\
\text { Secretary: Michael Johnston, Colgate } \\
\text { University } \\
\text { Program Chairman: Rei Shiratori, Institute } \\
\text { for Political Studies, Tokyo, Japan }\end{array}$ & $\begin{array}{l}\text { Newsletter (c/o Michael Johnston, } \\
\text { Colgate University) }\end{array}$ & $\begin{array}{l}\text { IPSA conferences and } \\
\text { irregular mid-term } \\
\text { roundtables }\end{array}$ \\
\hline $\begin{array}{l}\text { *THE SOCIETY FOR THE } \\
\text { PSYCHOLOGICAL STUDY } \\
\text { OF SOCIAL ISSUES } \\
\text { Lynda Fuerstnau, } \\
\text { Administrative Officer, P.O. } \\
\text { Box } 1248 \text {, Ann Arbor, MI } \\
48106-1248 \\
\text { Phone: (313) 662-9130 } \\
\text { Fax: (313) 662-5607 }\end{array}$ & $\begin{array}{l}\text { President: Sally Shumaker } \\
\text { President-Elect: Stuart Oskamp }\end{array}$ & $\begin{array}{l}\text { Journal of Social Issues, Daniel } \\
\text { Perlman, General Editor } \\
\text { Newsletter } \\
\text { SPSSI-sponsored volumes }\end{array}$ & $\begin{array}{l}\text { Midwest Executive } \\
\text { Meeting } \\
\text { APA convention }\end{array}$ \\
\hline $\begin{array}{l}\text { THE SOCIETY FOR THE } \\
\text { STUDY OF SOCIAL } \\
\text { PROBLEMS } \\
\text { 906 McClung Tower, } \\
\text { University of Tennessee, } \\
\text { Knoxville, TN } 37996 \\
\text { Phone: (615) } 974-3620 \\
\text { Fax: (615) } 974-7013\end{array}$ & $\begin{array}{l}\text { Executive Officer: Tom Hood } \\
\text { Administrative Officer: Michele Smith } \\
\text { Koontz } \\
\text { President: James Orcutt } \\
\text { President-Elect: Peter Conrad }\end{array}$ & $\begin{array}{l}\text { Social Problems, Robert Perrucci, } \\
\text { Purdue University, Editor } \\
\text { Newsletter, Richard Dello Buono, } \\
\text { Rosary College, Editor }\end{array}$ & \\
\hline $\begin{array}{l}\text { STATELESS NATIONS } \\
\text { SYMPOSIA AT THE } \\
\text { UNIVERSITY OF } \\
\text { WISCONSIN } \\
\text { P.O. Box 285, Brookfield, WI } \\
58008-0285\end{array}$ & $\begin{array}{l}\text { Board Members: Joseph Abramovich, } \\
\text { Vatro Murvar, Roland Stromberg }\end{array}$ & $\begin{array}{l}\text { Nation and Religion in Central } \\
\text { Europe and the Westem Balkans: } \\
\text { A Sociological Analysis } \\
\text { Submerged Nations: An Invitation to } \\
\text { Theory and Bibliography on One } \\
\text { Major Case Study (2nd ed. } \\
\text { forthcoming) } \\
\text { Program flyers } \\
\text { Newsletter } \\
\text { Available on request }\end{array}$ & $\begin{array}{l}\text { In conjunction with the } \\
\text { annual APSA, } \\
\text { AS(ociological)A } \\
\text { meetings and during the } \\
\text { academic year periodic } \\
\text { colloquia at the } \\
\text { University }\end{array}$ \\
\hline
\end{tabular}




\begin{tabular}{|c|c|c|c|}
\hline Organization & Officers & Publications & Meetings \\
\hline $\begin{array}{l}\text { TAFT UNIVERSITY } \\
420 \text { Lexington Ave., Suite } \\
\text { 2458, New York, NY } 10170 \\
\text { Phone: (212) 682-1530 } \\
\text { Fax: (212) } 953-1927\end{array}$ & $\begin{array}{l}\text { President: Maryann M. Feeney } \\
\text { Chairman: Prescott S. Bush, Jr. } \\
\text { Vice Chairmen: Hon. Birch E. Bayh, Jr. } \\
\text { William F. May }\end{array}$ & $\begin{array}{l}\text { Program flyers available on request } \\
\text { "Your Vote" video }\end{array}$ & $\begin{array}{l}\text { Annual Taft Seminars } \\
\text { for Teachers }\end{array}$ \\
\hline $\begin{array}{l}\text { *TRANSNATIONAL } \\
\text { STUDIES ASSOCIATION } \\
\text { John C. Di Pierro, Managing } \\
\text { Director, University of } \\
\text { Central Florida, P.O. Box } \\
\text { 25000, Orlando, FL } 32816\end{array}$ & $\begin{array}{l}\text { Managing Director: J. C.Di Pierro } \\
\text { Secretary-Treasurer: M. F. B. Garcia }\end{array}$ & $\begin{array}{l}\text { Global Perspectives: An } \\
\text { Interdisciplinary Joumal of } \\
\text { Intemational Relations } \\
\text { The TSA Report }\end{array}$ & $\begin{array}{l}\text { Irregular roundtables } \\
\text { and workshops } \\
\text { Annual meeting and } \\
\text { international conference }\end{array}$ \\
\hline $\begin{array}{l}\text { THE WALTER BAGEHOT } \\
\text { RESEARCH COUNCIL ON } \\
\text { NATIONAL } \\
\text { SOVEREIGNTY } \\
\text { 166-25 Powells Cove Blvd., } \\
\text { Beechhurst, NY } 11357\end{array}$ & $\begin{array}{l}\text { President and Editor-in-Chief: } \\
\text { Henry Paolucci } \\
\text { Vice President: Richard Charles Clark } \\
\text { Executive Director: Frank D. Grande, } \\
\text { City College of New York } \\
\text { Program Director: Frank Paul Le Veness, } \\
\text { St. John's University } \\
\text { Publications Coordinator: John H. Ryan, } \\
\text { Griffon House Press }\end{array}$ & $\begin{array}{l}\text { State of the Nation } \\
\text { Occasional papers } \\
\text { Special Proceedings } \\
\text { Foreign policy books }\end{array}$ & $\begin{array}{l}\text { In conjunction with the } \\
\text { APSA annual meeting, } \\
\text { in conjunction with NY } \\
\text { State Political Science } \\
\text { Association and } \\
\text { Northeastern Political } \\
\text { Science Association } \\
\text { meetings. Meetings at } \\
\text { campus centers. }\end{array}$ \\
\hline $\begin{array}{l}\text { WEBER (MAX) SYMPOSIA } \\
\text { AT THE UNIVERSITY OF } \\
\text { WISCONSIN } \\
\text { P.O. box } 285 \text {, Brookfield, WI } \\
53008-0285\end{array}$ & $\begin{array}{l}\text { Coordinator: V. Murvar, University of } \\
\text { Wisconsin-Milwaukee }\end{array}$ & $\begin{array}{l}\text { Nation and Religion in Central } \\
\text { Europe and the Western Balkans: } \\
\text { A Sociological Analysis } \\
\text { Max Weber Today: A Living Legacy } \\
\text { and Selected Bibliography } \\
\text { Program Flyers } \\
\text { Newsletter } \\
\text { Available on request }\end{array}$ & $\begin{array}{l}\text { In conjunction with the } \\
\text { annual APSA, } \\
\text { AS(ociological)A } \\
\text { meetings and during the } \\
\text { academic year colloquia } \\
\text { at the University of } \\
\text { Wisconsin }\end{array}$ \\
\hline $\begin{array}{l}\text { WESTERN SOCIAL } \\
\text { SCIENCE ASSOCIATION } \\
\text { Bryan T. Downes, Executive } \\
\text { Director, Dept. of Planning, } \\
\text { Public Policy \& Management, } \\
\text { University of Oregon, } \\
\text { Eugene, OR } 97403 \\
\text { Phone: (503) } 346-3817 \\
\text { Fax: (503) 346-2040 }\end{array}$ & $\begin{array}{l}\text { President: N. Joseph Cayer, Arizona State } \\
\text { University } \\
\text { President-Elect: Edward Glatfelter, } \\
\text { Utah State University } \\
\text { Vice President: Jerry Polinard, University } \\
\text { of Texas, Pan American } \\
\text { Past-President: Andrew Schoolmaster, } \\
\text { University of North Texas }\end{array}$ & $\begin{array}{l}\text { Social Science Joumal, David A. } \\
\text { Freeman, Washburn University, } \\
\text { Editor } \\
\text { WSSA News (newsletter), Laurence } \\
\text { Shute, California State University, } \\
\text { Pomona, Editor }\end{array}$ & $\begin{array}{l}\text { Annual conference } \\
\text { April 26-29, } 1995 \\
\text { Oakland, CA }\end{array}$ \\
\hline $\begin{array}{l}\text { WOMEN'S CAUCUS IN } \\
\text { POLITICAL SCIENCE, } \\
\text { Department of Public and } \\
\text { International Affairs } \\
\text { George Mason University } \\
\text { Fairfax, VA 22030-4444 } \\
\text { Phone: (703) 993-8718 }\end{array}$ & $\begin{array}{l}\text { President: Toni-Michaelle Travis, George } \\
\text { Mason University } \\
\text { President-Elect: Jane Mansbridge, } \\
\text { Northwestern University } \\
\text { Secretary: Emily Gill, Bradley University } \\
\text { Treasurer: Maren Stein, Russell Sage } \\
\text { College } \\
\text { Membership Secretary: Laine Kosaki, Beloit } \\
\text { College }\end{array}$ & $\begin{array}{l}\text { WCPS Quarterly, Cynthia Duguette, } \\
\text { Wayne State University }\end{array}$ & $\begin{array}{l}\text { At APSA Annual } \\
\text { Meeting }\end{array}$ \\
\hline
\end{tabular}

